\title{
A case of infertility with tuberculosis with multiple calcifications on both fallopian tubes
}

\author{
Meena Khatri*, Preshit Chate
}

Department of Obstetrics \& Gynecology, Jawaharlal Nehru Medical College, AVBRH, Sawangi, Meghe, Wardha, Maharashtra-442002, India

Received: 18 January 2013

Accepted: 23 January 2013

*Correspondence:

Dr. Meena Khatri

E-mail: meenakhatri619@yahoo.com

\begin{abstract}
Genital tract tuberculosis usually shows no symptoms and it is diagnosed as such incidentally during infertility investigations. In general, infertility is the most common clinical symptom of pelvic tuberculosis to the extent that it constitutes between 40 to 60 percent of the patients chief complaints. Female genital tuberculosis is still a major cause of infertility in India. This case highlights some of the well known facts about genital tuberculosis, that it is the relatively young female in the reproductive age group who are victim in female genital tuberculosis and infertility. In our case inspite of advanced genital tuberculosis, endometrial biopsy was negative for AFB and showed normal histopathology and diagnostic laparoscopy and biopsy from fallopian tubes revealed genital tuberculosis. Thus laparoscopy has definite place in the diagnosis of pelvic tuberculosis. Female genital tuberculosis is a major cause of infertility however diagnosis is sometime difficult and it becomes still more difficult when endometrial histopathology is normal therefore diagnostic laparoscopy is gold standard for confirmation of genital tuberculosis with its added advantages of complete evaluation of uterus, tubes, ovaries and operative intervention in the same sitting.
\end{abstract}

Keywords: Infertility, Genital tuberculosis, Calcification of tubes, Laparoscopy

\section{INTRODUCTION}

Genital tract tuberculosis usually shows no symptoms and it is diagnosed as such incidentally during infertility investigations. In general, infertility is the most common clinical symptom of pelvic tuberculosis to the extent that it constitutes between 40 to 60 percent of the patients chief complaints. ${ }^{1}$

\section{CASE REPORT}

A 23 years old woman admitted from infertility clinic with chief complaints of primary infertility since 7 years, menorrhagia since 3-4 months, dyspareunia since 3-4 months, her previous menstrual cycles were regular 3 4/28 days but since 3-4 months, her cycles were 7-8/28 days. Her vital parameters were within normal limit, abdomen soft. On speculum examination; cervix and vagina were healthy. Per vaginal examination: uterus normal size, retroverted freely mobile, fornices clear.
Her haemoglobin was $11.5 \mathrm{gm} \%$, TLC $12500 / \mathrm{mm}^{3}$, P$76 \%, \mathrm{~L}-20 \%, \mathrm{E}-3 \%, \mathrm{M}-2 \%$, ESR $65 \mathrm{~mm} 1^{\text {st }}$ hour, X-ray chest NAD, Pelvic USG within normal limit and mantoux test negative. ELISA test TBIgG-180U/ml, TB IgM$0.95 \mathrm{U} / \mathrm{ml}$ (equivocal). Husband's investigations were found within normal limit.

Diagnostic laparoscopy: Showed uterus covered with multiple tubercles and calcifications, one big calcified nodule seen on posterior wall of uterus on left side (Figure 1). Both fallopian tubes were dilated at distal ends and showing linear calcifications (Figure 2), pelvic peritoneum studded with tubercles but no free fluid in pelvis, biopsy taken from tubercles and fallopian tubes and tissue sent for histopathological diagnosis also D\&C done and endometrium sent for histopathology and AFB culture. 


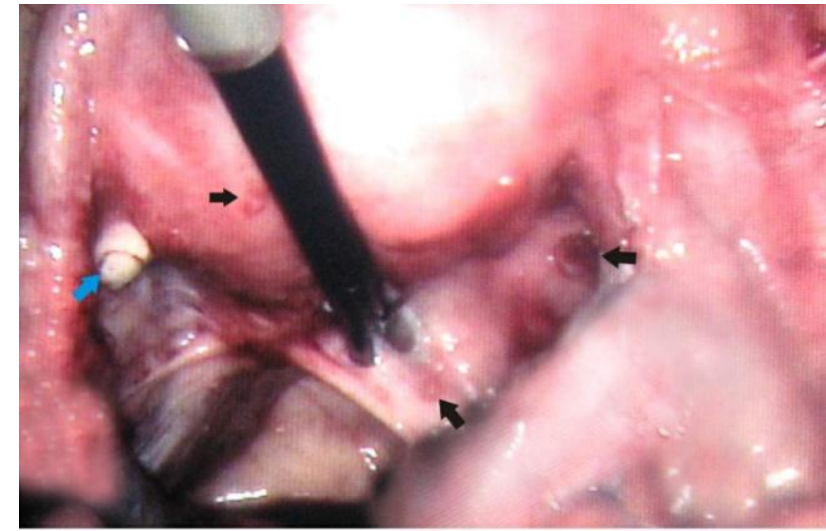

Figure 1: Arrow showing big calcified nodule on posterior wall of uterus on left side.

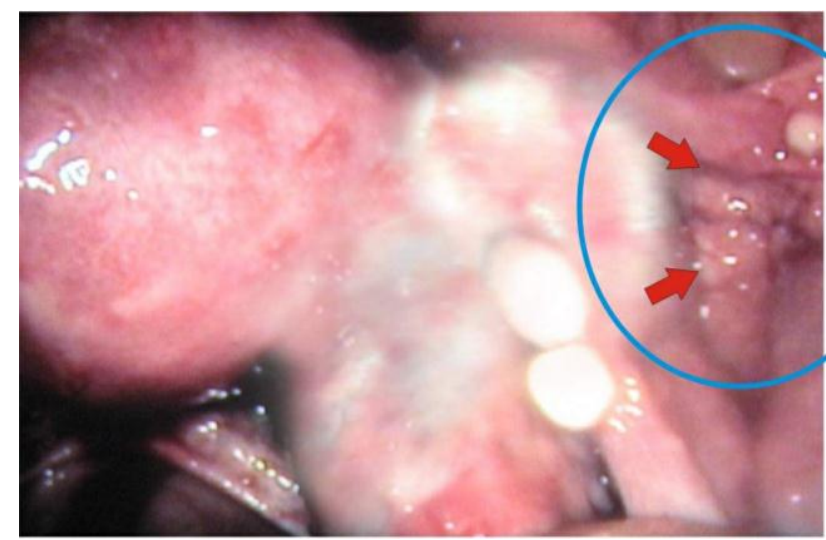

Figure 2: Grossly dilated fallopian tube with linear calcification.

Histopathology report of biopsy from tubercles and tubes: Suggestive of tubercular granuloma (Figure 3), while endometrium was within normal limit, AFB culture- sterile.

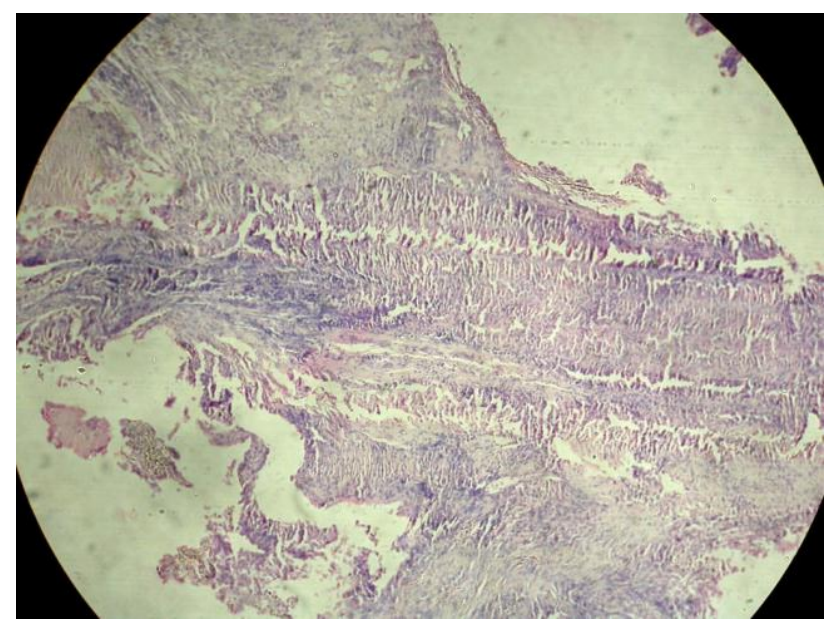

Figure 3: Microphotograph showing tubercular granuloma.
Patient was started with category I extrapulmonary antitubercular regime: 2(HRZE) $3+4(\mathrm{HR}) 3$, i.e. INH-600 $\mathrm{mg}$, Rifampicin 450mg, Pyrazinamide 1500mg, Ethambutol $1200 \mathrm{mg}$, thrice weekly and she was advised to come for follow up.

\section{DISCUSSION}

Female genital tuberculosis is still a major cause of infertility in India. ${ }^{2}$ This case highlights some of the well known facts about genital tuberculosis, that it is the relatively young female in the reproductive age group who are victim in female genital tuberculosis and infertility. ${ }^{3}$ Both fallopian tubes are involved in almost all patients with pelvic tuberculosis and infertility is the most common clinical symptom of pelvic tuberculosis even if fallopian tubes are not obstructed. ${ }^{4,5}$ The finding of endometrial tuberculosis almost always means that the tubes are infected but tubercular salpingitis is sometimes found without associated endometritis in 30-40 percent of cases $^{6}$ and a real problem is to diagnose tubal tuberculosis in the absence of endometrial involvement. In our case inspite of advanced genital tuberculosis, endometrial biopsy was negative for AFB and showed normal histopathology and diagnostic laparoscopy and biopsy from fallopian tubes revealed genital tuberculosis. Thus laparoscopy has definite place in the diagnosis of pelvic tuberculosis.

\section{CONCLUSION}

Female genital tuberculosis is a major cause of infertility however diagnosis is sometime difficult and it becomes still more difficult when endometrial histopathology is normal therefore diagnostic laparoscopy is gold standard for confirmation of genital tuberculosis with its added advantages of complete evaluation of uterus, tubes, ovaries and operative intervention in the same sitting.

\section{ACKNOWLEDGEMENTS}

We acknowledge our gratitude towards Anesthetist and Pathologist for helping us to complete the case report and grateful to patient permitting to publish this case report.

Funding: No funding sources

Competing interests: There are no competing interests to declare

Ethical approval: Not required

\section{REFERENCES}

1. Namavar Jahromi B, Parsanezhad ME, Ghane-shirazi R. Female Genital tuberculosis and infertility. Int J Gynaecol Obstet 2001;75:269-72.

2. Rozati R, Sreenivasagari R, Cheruvu R. Evaluation of women with infertility and genital tuberculosis. J Obstet Gynaecol India 2006;56:423-6.

3. Tripathy SN. Genital affection in pulmonary TB. Indian J Tuberc 1991;38:191-6. 
4. Chavhan GB, Hira P, Rathod K, Zacharia TT, Chawla A, Badhe $\mathrm{P}$, et al. Female genital tuberculosis: hysterosalpingographic appearances. $\mathrm{Br}$ J Radiol 2004;77:164-9.

5. Hatami M. Tuberculosis of female genital tract in Iran. Arch Iran Med 2005;8:32-5.
6. Bapna N, Swarnakar M, Kotia N. Genital tuberculosis and its consequences on subsequent fertility. J Obstet Gynaecol India 2005;55:534-7.

DOI: $10.5455 / 2320-1770 . i j r \operatorname{cog} 20130223$

Cite this article as: Khatri M, Chate P. A case of infertility with tuberculosis with multiple calcifications on both fallopian tubes. Int J Reprod Contracept Obstet Gynecol 2013;2:111-3. 